\title{
Estimating the Effect of Asymmetric Electrodes in Bipolar Discharge Ion Wind Generator
}

\author{
Van Thanh Dau \\ Sumitomo Chemical. Ltd., Research Group (Environmental Health), \\ Hyogo 665-8555, Japan \\ Canh-Dung Tran \\ University of Southern Queensland, School of Mechanical and Electrical Engineering \\ QLD 4350, Australia \\ and \\ National University of Singapore \\ Department of Mechanical Engineering, Singapore \\ Thien Xuan Dinh \\ Ritsumeikan University, Graduate School of Science and Engineering, \\ Shiga 525-8577, Japan \\ Lam Bao Dang \\ Hanoi University of Science and Technology \\ Viet Nam \\ Tibor Terebessy \\ Atrium Innovation Ltd., Lupton Road, OX10 9BT \\ Wallingford, United Kingdom \\ Tung Thanh Bui \\ Vietnam National University, University of Engineering and Technology \\ Hanoi, Vietnam
}

\begin{abstract}
We present the study of a simple and efficient air-flow generator under the effect of ion winds which are created by corona discharge. Based on our recent research of ion wind with bipolar corona using parallel pins, this work reports an investigation and evaluation of the corona system in asymmetrical configurations, where the two electrodes have different tip shapes. The system of new configuration is investigated both experimentally and numerically. The numerical and experimental results are the base to establish a regime of stable operation by the measured I-V characteristics. The optimized set-up can generate an air flow up to $2.24 \mathrm{~m} / \mathrm{s}$ at the atmospheric pressure. With the asymmetrical configuration of different pin tip shapes, the system can be used in many mixing applications such as mixing solvent and die where the generated vortices are also presented.
\end{abstract}

Index Terms - electrohydrodynamics, asymmetric electrodes, air-flow generator, vortex.

\section{INTRODUCTION}

SINCE the pioneering work on ion wind generated by

Manuscript received on 26 June 2017, in final form 7 March 2018, accepted 7 March 2018. Corresponding author: Van T. Dau. corona discharge from Chattock [1] in 1899, a great number of ion wind applications have been published in various fields including propulsion technology [2-5], micropump design [610], cooling applications [11-13], and gas spectrometry [14, 15]. There are several different configurations used in ion wind generators such as point - plane [16], point - grid [17], point - 
ring [18], wire - plate [19], wire - inclined wing [20] or wire non parallel plates [21], in which a sharp electrode generates ionized species under a strong electric field. The most common configurations include the point - plane and point - ring where ions are created in the vicinity of the sharp electrode also referred to as pin. The reference electrode is placed downstream from the pin tip and defines the movement of charged particles along the electric field lines. For certain applications, it is necessary to install an additional neutralizing component or to introduce a device cover grounded in order to protect the system from charges, but that leads to more complicated design. Such problems have been solved using a symmetrical configuration [22-28] where two electrodes with identical tips are placed in parallel and both generate charged particles. In this configuration, the generated ion wind movement is in parallel with the pins.

Among the recently established applications of ion wind, the micro sample transporting and mixing are promising. For example, the asymmetric ion wind offers an efficient solution to create and manipulate micro vortices which is a potential way to transport micro particles and concentrate them to a predetermined location [29, 30]. Dunn-Rankin et al [31] created swirl flow in an earthed cylinder by circularly arraying four pin discharge electrodes around its center. Yeo et al [32, 33] have used ion wind generated between a needle and a transparent electrode to create micro vortices which can be applied to increase the concentration of biological samples and shorten the cultivation time [34]. A recent study by Zhao et al [35] suggested the use of dual pins parallel to a grounded plate to generate vortices. For this configuration, an asymmetric ion wind is created when pins are connected to different voltage levels. Since the obtained ion winds move toward the plate with different velocities, they generate vortices. Obviously, in these unipolar discharge systems, the target is considered as a part of the high voltage electric circuit to form corona discharge. In the corona induced vortex, since the target is also ground, some materials are not applicable [32-35]. In addition, it has been seen that the highly reactive ions released by unipolar discharge plasma can easily penetrate into a liquid and then significantly modify its composition [36]. If the target chip is a component of high voltage circuit, accumulated particles may generate electric field singularities to ignite the discharge on the chip and ultimately damage the bio-targets $[37,38]$.

In this paper, we study a compact and simple ion wind generator using two parallel pin electrodes whose tips have different shapes. Such configurations are called as asymmetric ones. Since ion winds are generated by the electrodes themselves without the necessity of a grounded target, this approach is more flexible than the systems mentioned above. Therefore, the target can be made using any kind of materials including plastic, glass, gel, metal or semiconductor. Because of the asymmetry of configuration, the intensity and direction of ion winds can be easily controlled. In addition, for the first time by the present system, the current and voltage (I-V) characteristics can be analyzed to pinpoint the general relation between the $\mathrm{I}-\mathrm{V}$ and the asymmetry. Analyses of the experimental I-V relationship by this work has found an unstable discharge operation with inefficient ion wind generation. That yields an efficient method to design an optimized system for a given configuration such as the geometrical constraints (electrodes' inter-distance) and satisfying system requests, for example the velocity of ion wind based on the analysis of obtained I-V characteristics.

For the sake of following experiments easily, we classify the pin shape of electrodes by their sphere radius (SR) as follows: the electrode of sharp tip with $\mathrm{SR}=15 \mu \mathrm{m}$, conical tip with SR $=80 \mu \mathrm{m}$ and round tip with $\mathrm{SR}=400 \mu \mathrm{m}$. Also, the configuration of a system is defined either symmetrical or asymmetrical one if its electrodes' shapes are similar or different from each other, respectively. In our previous works, ion wind of generator whose electrodes are the same with sharp or conical tip has been investigated [22]. In this paper, asymmetric configurations of the combination of three pin tip types SR15(+)/SR80(-), SR15(-)/SR80(+), SR15(+)/SR400(-) and SR15(-)/SR400(+), where the signs $(+)$ and (-) indicate voltage polarity will be studied. The I-V characteristic of the corona discharge is recorded and then analyzed for each individual configuration. The induced ionic wind is investigated by both experimental and simulation works. Furthermore, the use of the new approach and device for sample mixing is also carried out and evaluated.

\section{EXPERIMENTAL SETUP}

The ion wind generator includes two stainless steel electrodes of $8 \mathrm{~mm}$ length and $0.4 \mathrm{~mm}$ diameter placed in parallel with each other as shown in Fig. 1a. The electrodes are connected to a high voltage generator which can generate a range of $10 \mathrm{kV}$.

(a)

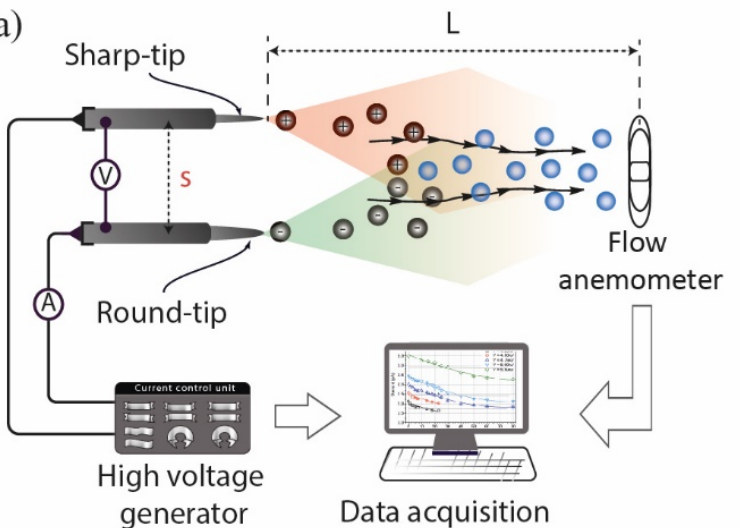

(b)

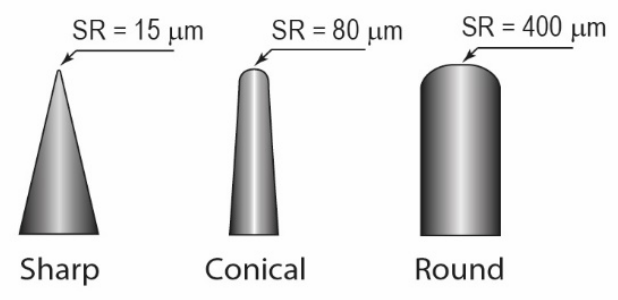

Figure 1. Set-up of the experiment; (a) schematic of the system and (b) the geometry of SR electrode tips used in the experiment.

The system of different configurations using the combination of three different electrodes including sharp (SR15), conical (SR80) and round tips (SR400) is 
investigated (Figure 1b). The distance $s$ between the electrodes is changeable using a three-axis movable stage. As the experiment is carried out with very low currents but high voltages, the electrodes need to be specially protected by an insulation with resistance values exceeding $100 \mathrm{GOhm}$ in order to minimize the leak current. A thermal anemometer (ISA-90N) is installed $\mathrm{L}=25 \mathrm{~mm}$ downstream of the device to monitor the ion wind velocity. The anemometer is placed in the vertical plane $25 \mathrm{~mm}$ away from the electrode tip. In this plane, the anemometer is set up at the same height with one of the electrodes. Because of the position where the velocity reaches its peak value depends on the applied voltage and/or the pin electrode configuration, the anemometer is carefully adjusted to a suitable position on its plane to obtain the measured peak velocity.

\section{CURRENT - DISCHARGE VOLTAGE CHARACTERISTICS}

In our previous works [23], we reported that in the symmetrical bipolar systems, the I-V characteristics expressed by the relation $\sqrt{\mathrm{I}} \propto \mathrm{V}$ is in better agreement with the experimental results in comparison with the traditional empirical Townsend relation.

Figure 2 describes the experimental relationship between current and voltage in the form of $\sqrt{I} \propto V$. The results show

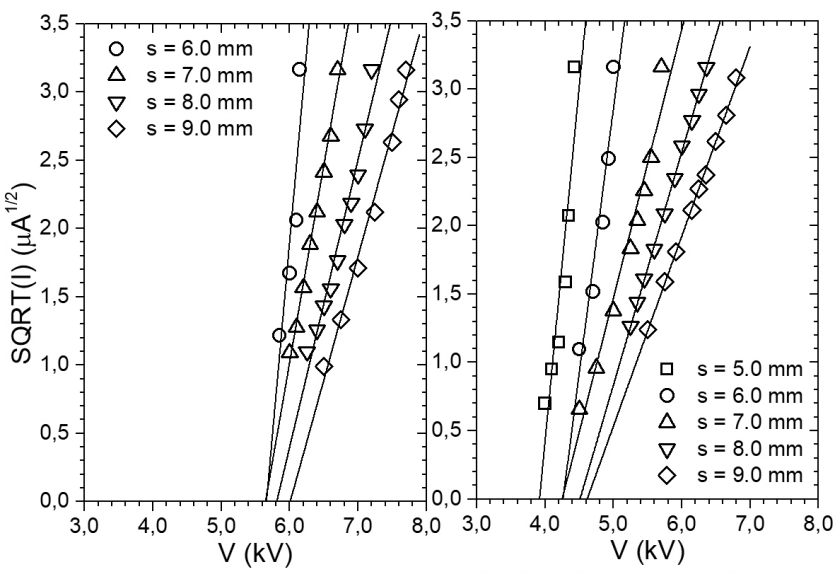

(a) SR400(-) and SR15(+)

(b) SR400(+) and SR15(-)

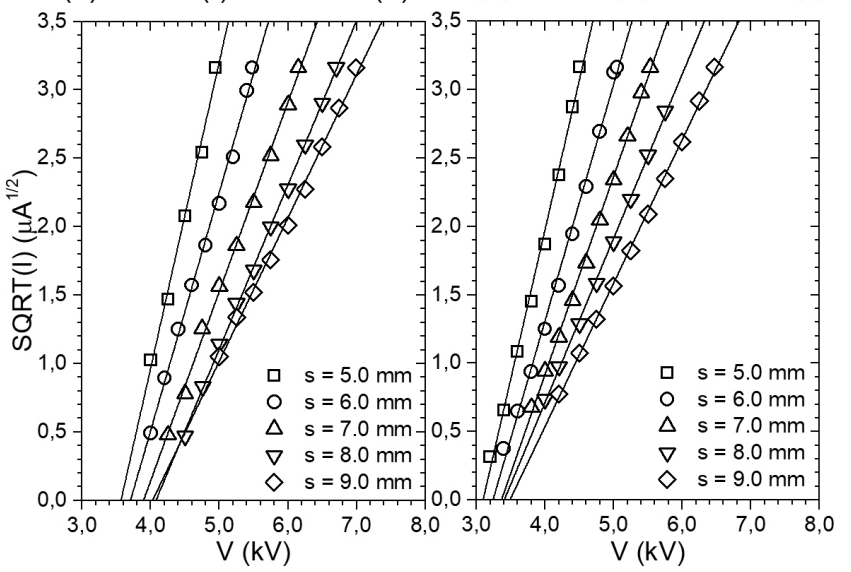

(c) SR80(-) and SR15(+)

(d) SR80(+) and SR15(-)

Figure 2. $\mathrm{I}-\mathrm{V}$ characteristics of asymmetric configurations using various shapes of pin tip with different inter-electrode distances. that the corona discharge starts with the highest onset voltage for the configuration using round pin as either positive or negative electrode. The onset voltage is higher for less sharp pin used as a negative electrode. The obtained data evidence that depending on the set of factors including shape of electrode pins, inter-electrode distance and voltage distribution on electrodes, the asymmetrical configuration of the present system is considered as point - plane, bipolar or a combination of them. An interpretation evidenced by experimental results is given as follows.

In the bipolar regime, both electrodes generate ion winds of opposite charges. The resulting ion winds will impinge on each other within the interelectrode space to generate a steady flow moving away from the device. The ions of opposite charges quickly recombine with each other, hence, the majority of ions generated at both electrodes will not reach the counter electrode [24]. The configuration of SR15(+)/SR400(-) of this research with I-V characteristic given in Fig 2a operates by the bipolar regime. In fact, until the onset voltage reached a strong value $\left(\mathrm{V}_{0}=5.8 \mathrm{kV}\right.$ in this work), both electrodes of sharp and round pins, SR15(+) and SR400(-), respectively, are not ready to release their ion winds. This is confirmed by corona observation under microscope and the resulting discharge is a bipolar with stable strong ion wind as presented in Figure 5a.

On the other hand, in a point - plane regime, one of the

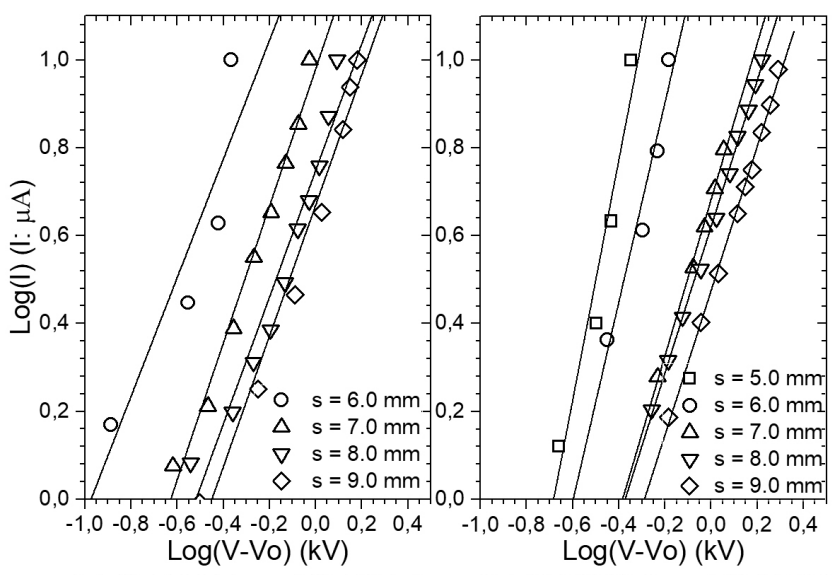

$\begin{array}{ll}\text { (a) SR400(-) and SR15(+) } & \text { (b) SR400(+) and SR15(-) }\end{array}$

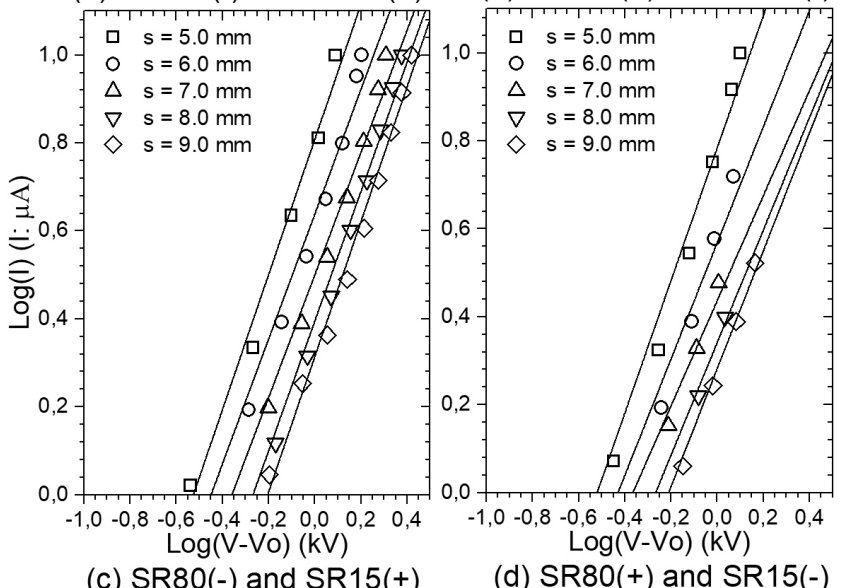

(c) SR80(-) and SR15(+)

(d) SR80(+) and SR15(-)

Figure 3. Relation of $\log (\mathrm{I})$ and $\log (\mathrm{V}-\mathrm{V} 0)$ plotted for each configuration with different inter-electrode distances. 
electrodes is capable of generating corona while the other one is not as it processes a weaker electric field than discharge one. This can be observed for the configuration with the I-V characteristic shown in Figure $2 b$ where the negative electrode of sharp tip starts early releasing charges at voltage of $4 \mathrm{kV}$. Since the system is closed and balanced, the negative ion wind tends to move to the positive electrode because of lacking sufficiently strong opposite charge. Thus, in this phase, the configuration SR15(-)/SR400(+) operates as a point - plane one with the sharp electrode SR15(-) as a point and the round one $\operatorname{SR} 400(+)$ as a plane. Until the voltage increases up to an enough strong value, the positive electrode of round pin also generates charges as the sharp electrode does and the system operates as a bipolar one.

The experiment using mixed configurations with electrodes of conical and sharp pins (Figures 2c-d) reveals that the charge is released from both pins. Results show the resulting discharge is stable with relatively strong ion wind at a sufficiently strong voltage and the system works like a bipolar one. Although the discharge occurs by a regime of the combination of bipolar and point - plane configurations with weaker ion wind at a lower voltage, this phase is quickly diminished with the rising of voltage.

In order to investigate the relationship between the I-V characteristic and the discharge stability, the characteristic in the general form of $\mathrm{I}=\mathrm{C} \mu_{\mathrm{i}}\left(V-V_{0}\right)^{n}$ is introduced in the decimal logarithmic scale as follows:

$$
\log (\mathrm{I})=\mathrm{n} \times \log \left(\mathrm{V}-\mathrm{V}_{0}\right)+\log \mathrm{C} \mu_{\mathrm{i}}
$$

where $n$ and $\mathrm{C}$ are coefficients, which depend on the geometry, material and configuration of electrodes; $\mu_{\mathrm{i}}=$ $\mu_{0}=2 \times 10^{-4} \mathrm{~m}^{2} V^{-1} s^{-1}$ is the mobility of ions in the atmosphere at standard condition and $V_{0}$ is the onset voltage indicating the corona burst and determined by an abrupt change of current. The onset voltage is usually selected at a current below $1 \mu \mathrm{A}$, as suggested by Lama and Gallo [39]. It is noted that there may be two different voltage onsets, with either similar of significantly different values, corresponding to the bipolar and unipolar discharge systems, respectively. Indeed, the relation between $\mathrm{I}$ and $\left(\mathrm{V}-\mathrm{V}_{0}\right)^{n}$ is covered over a large range of experimental data with a very small current $\mathrm{I}=0.1 \mu \mathrm{A}$ chosen in this work.

Figure 3 presents the linear relations between $\log (\mathrm{I})$ and $\log \left(\mathrm{V}-\mathrm{V}_{0}\right)$ with onset voltages at the current $\mathrm{I}=0.1 \mu \mathrm{A}$ for a range of inter-electrode distances. While the physical spaces of $\mathrm{I}$ and $(\mathrm{V}-\mathrm{Vo})$ are $\left(\mathrm{I}_{0},+\infty\right)$ and $(0,+\infty)$, respectively, the value fields of $\log (\mathrm{I})$ and $\log (\mathrm{V}-\mathrm{Vo})$ in Fig. 3 indicate both corresponding ranges of $\mathrm{I}$ and $\mathrm{V}-\mathrm{Vo}$ covering $(-\infty,+\infty)$. This advantage of the $\left(V-V_{0}\right) \propto I^{1 / n}$ characteristic allows to overcome the common difficulties of using equation I $\propto$ $\mathrm{V}\left(\mathrm{V}-\mathrm{V}_{0}\right)$ where the onset voltage by an extrapolation sometimes gains a negative value without physical meaning [40].

Figure 4 presents the exponent $n$ extracted from Equation (1), plotted as a function of inter-electrode distance with different configurations. It can be seen that except with the configuration $\mathrm{SR} 400(+) / \mathrm{SR} 15(-)$, the exponents $n$ in the other configurations are nearly the same and vary from 1.3 to 1.6 , which are similar to ones by the symmetrical bipolar discharge published in our recent work [23].

It is worth noting that the electrodes SR80 and SR15 can efficiently generate ion winds for both polarities (SR80 $(+/-$ )/SR15(-/+)), thus the exponents $n$ in SR80(+/-)/SR15(-/+) would be the same and similar to those observed in symmetrical configurations. In addition, since the negative corona is easily generated even by electrode of round tip, the configuration SR400(-)/SR15(+) can also be considered as a bipolar discharged system. Thus, its exponent $n$ is similar to those of the configuration SR80(+/-)/SR15(-/+), and does not depend on the inter-electrode distance [28]. Meanwhile, it is harder to generate corona discharge by an asymmetric configuration with positive electrode of round tip because positive corona requires a pretty high onset voltage [41]. This can explain why the exponent $n$ by the configuration SR400(+)/SR15(-) is very different from the others as presented in Figure 4.

Interestingly, experimental results on ion wind in Figure 5 show that SR400(+)/SR15(-) is not an efficient configuration used in generators. In fact, since corona by SR400(+) is unstable, the configuration is likely a point plane one where more charges come from the negative electrode SR15(-), especially when this electrode tip size is considerable compared with the inter-electrode distance $s$. For example, the exponent $n$ is much higher for $s=5-7 \mathrm{~mm}$ as depicted in Figure 4. In addition, with a sufficiently large inter-electrode distance, the difference between electrode tips becomes insignificant and the exponent $n$ gradually reduces and reaches to 1.5 . This observation is evidenced by experimental results of ion wind presented in Figure 5 in which the velocity of ion wind by the configuration SR400(+)/SR15(-) is different from those by other configurations for small inter-electrode distances but almost the same for large ones.

Thus, the exponent $n$ is a reliable indicator to predict whether an asymmetric configuration is close to a point plane or bipolar regime. The results of $n$ obtained by our

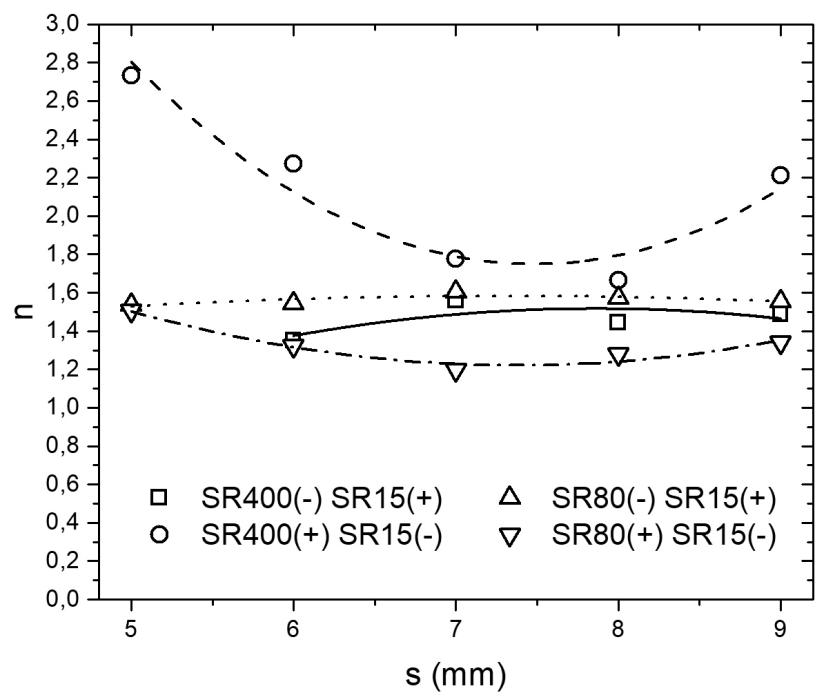

Figure 4. Exponent $\mathrm{n}$ of $\mathrm{I}-\mathrm{V}$ characteristics plotted with different interelectrode distances using the current of $0.1 \mu \mathrm{A}$. 
present work are in good agreement with those by other publications using similar inter-electrode distances [16], [40], [42]. In summary, since a sufficiently high value of $n$, an asymmetric configuration operates with point - plane regime and ion wind generated by an electrode mainly moves towards another one, while the system with lower exponent $n$ works with bipolar regime and ion winds move away from electrodes. This idea will be further studied with sufficient details in our future work.

\section{EFFECT OF CONFIGURATION PARAMETERS ON THE ION WIND VELOCITY}

In this section, the ion wind generated at different discharge powers is studied. Experimental results by Figure $5 a-d$ show that the ion wind velocity increases with the increase of the discharge power $(\mathrm{V} \times \mathrm{I})$. The increase rate of ion wind velocity is reduced and the velocity tends to reach a stable value as observed in Figures 5 a-d.

It can be seen that the inter-electrode distance also affects the velocity of ion wind for any configuration as described in Figures 5a-d. In general, the ion wind velocity initially decreases with the reduction of the inter-electrode distance for all considered configurations. This can be explained as with decreasing distance, the resistance caused by the

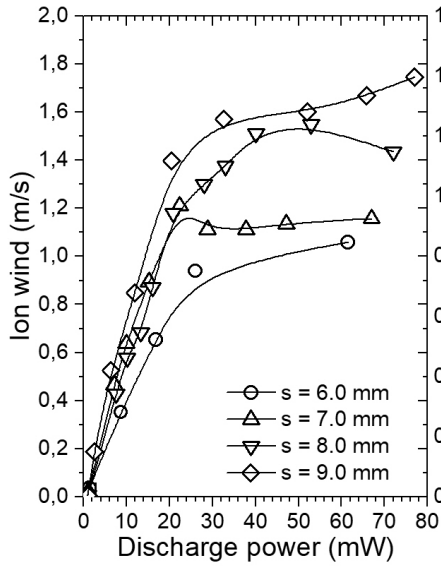

(a) SR400(-) and SR15(+)

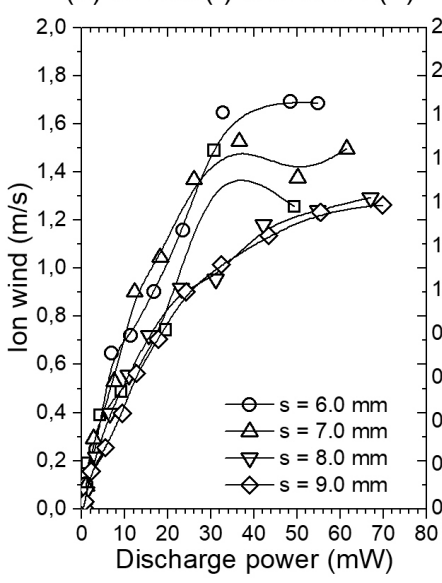

(b) SR400(+) and SR15(-) (c) SR80(-) and SR15(+)

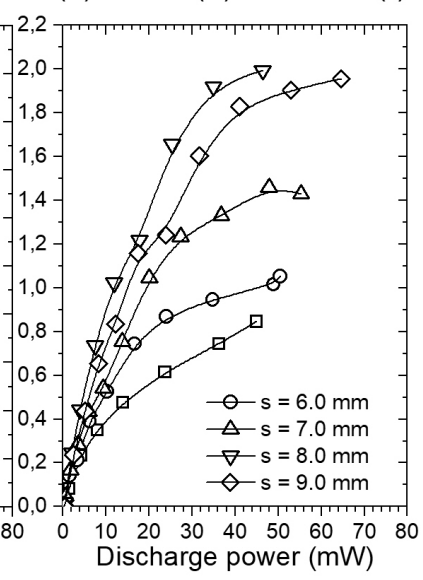

(d) SR80(+) and SR15(-) electrodes on the ion wind movement becomes more significant regardless of the shape of pin tip. Moreover, the decrease in electrode gap causes the impingement of ion jets at a steeper angle, resulting in more momentum loss and reduction of total flow.

However, for the electrodes of conical pin tip SR80 given in Figure $5 \mathrm{c}$ and $\mathrm{d}$, the velocity of ion wind reaches a maximum value with a relevant gap of electrodes and decrease with the increase of the inter-electrode distance. For example the velocities of configurations SR80(+)/SR15(-) (Figure 5c) and SR80(-)/SR15(+) (Figure 5d) reaches to optimum values with the inter-electrode distances $\mathrm{s}=6.0 \mathrm{~mm}$ and $8.0 \mathrm{~mm}$, respectively.

Also, it is noticeable that a configuration using electrode of conical pin (SR80) generates a stronger ion wind compared with one using electrode of round pin tip (SR400) as observed in Figure 5c versus Figure 5a. Moreover, a system using round electrode connected to negative voltage produces ion wind of stronger velocity. For example, the velocity of ion wind generated by the configuration SR400()/SR15(+) (Figure 5a) is stronger than those with SR400(+)/SR15(-) (Figure 5b) with every inter-electrode distances. Meanwhile, for configurations with electrode of conical pin tip connected to positive voltage, SR80(+)/SR15(-) (Figure 5d), the velocity of ion wind generated is stronger than one by SR80(-)/SR15(+) (Figure $5 \mathrm{c})$. For example, the highest velocity of ion wind achieved $2.24 \mathrm{~m} / \mathrm{s}$ with SR80(+)/SR15(-), higher than one of SR80()/SR15(+) (about $1.7 \mathrm{~m} / \mathrm{s}$ presented in Figure $5 \mathrm{c}$ ).

These results are in agreement with the two discharge regimes identified in the previous section. As discussed, a configuration with positive electrode of round pin tip cannot generate sufficient ion wind because the system behaves as point - plane regime. The ion wind by an electrode is then directed towards its counter electrode, yields a decrease of the ion wind's strength in overall. Meanwhile a configuration with electrode of conical pin tip induces the sufficient corona discharge for both electrodes (bipolar regime), which is evidenced by experimental results described in Figure 4.

\section{NUMERICAL SIMULATION, DISCUSSION AND APPLICATION}

Numerical studies of ion wind generator have been presented for most ion wind configurations [43-48]. In this work, a simplified charge transport model for the discharge drift area is used with the boundary condition of charge density at the tips of electrodes given by the measured current-voltage $(\mathrm{I}-\mathrm{V})$ characteristics of the corona to simulate the motion of ion wind. For the steady state, the air motion under the interaction of electric field is governed by the following equations:

$$
\begin{aligned}
& \nabla \cdot\left( \pm \mu \vec{E} \rho_{ \pm} \pm \vec{U} \rho_{ \pm}\right)=-R_{i} \rho_{+} \rho_{-} / q_{e} \\
& \nabla \cdot(\nabla \varnothing)=-\left(\rho_{+}-\rho_{-}\right) / \varepsilon_{0} \\
& \nabla \cdot(\vec{U} \vec{U})-\nabla \cdot(\nu \nabla \vec{U})=-\nabla p+\left(\rho_{+}-\rho_{-}\right) \vec{E} / \rho \\
& \nabla \cdot \vec{U}=0
\end{aligned}
$$

Figure 5. Velocity of ion wind at different inter-electrode distances. 


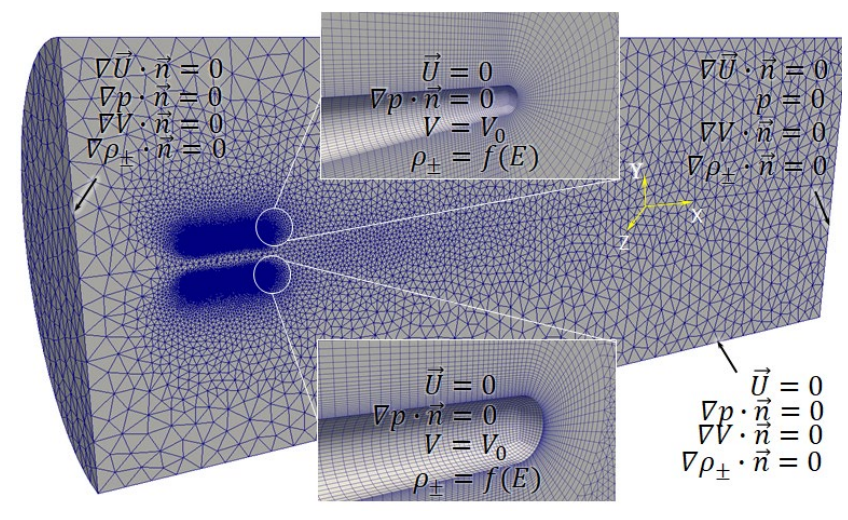

Figure 6. Simulation setup, meshing and boundary conditions.

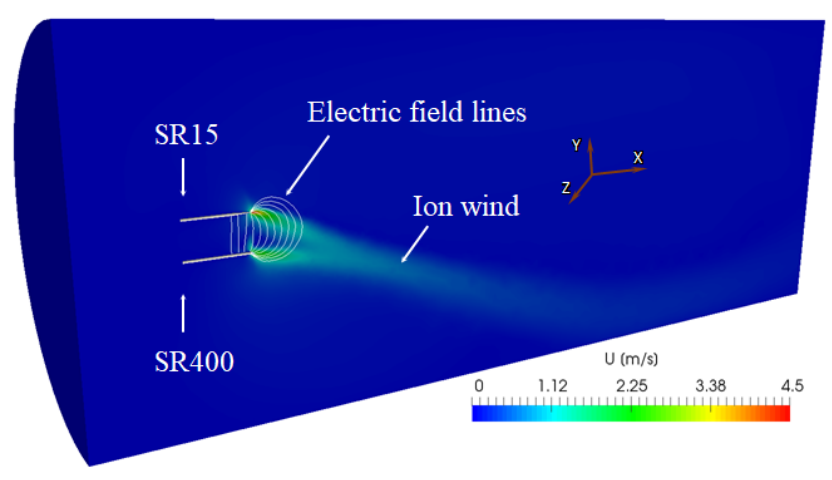

Figure 7. Simulation result, asymmetric flow of ion wind generated by the asymmetric configuration SR400/SR15.

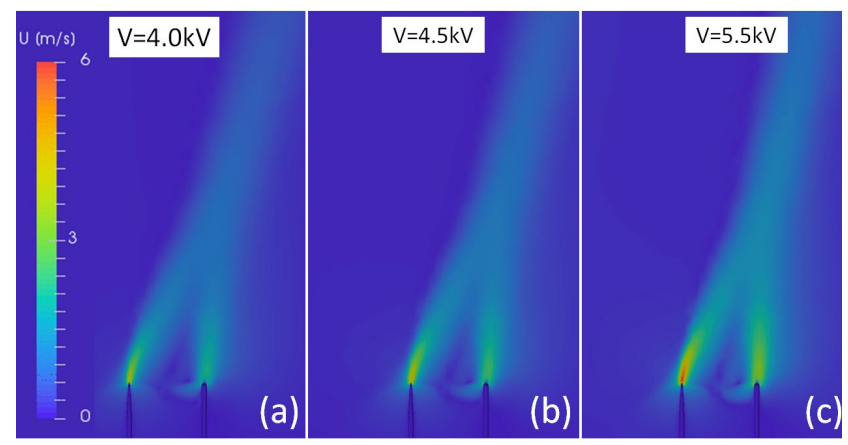

(d) 1,5

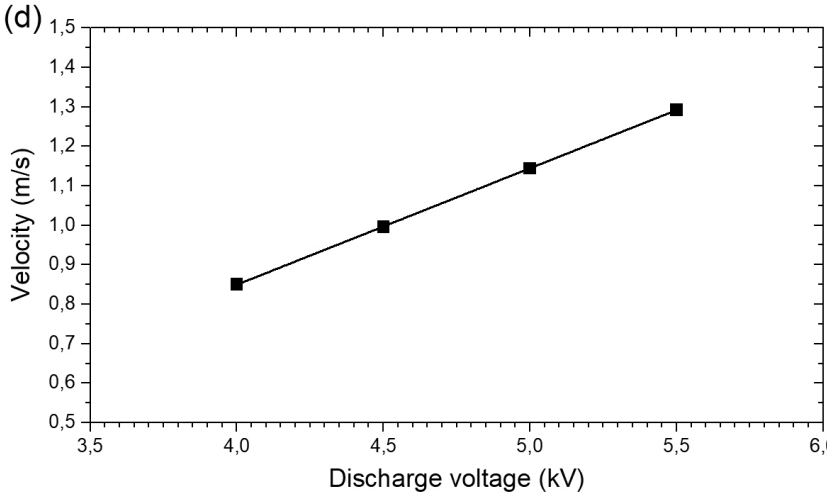

Figure 8. Relationship between the ion wind velocity and discharge voltage by numerical simulation with inter-electrode distance of $8 \mathrm{~mm}$ using the configuration SR15/ SR400.

$$
\rho_{ \pm}=i_{\mathrm{exp}} /\left(\mu E_{\mathrm{on}} A\right)
$$

where $\rho_{+}$and $\rho_{-}$are the charge densities of positive and negative ions of the current density mainly caused by the ion drift $\left(\mu \vec{E} \rho_{ \pm}\right)$and ion convection $\left(\vec{U} \rho_{ \pm}\right), \mu=1.6 \times$ $10^{-4} \mathrm{~m}^{2} \cdot \mathrm{V}^{-1} \cdot \mathrm{s}^{-1}$ is the mobility of positive and negative charges, $R_{i}=10^{-13} \mathrm{~m}^{3} \cdot \mathrm{s}^{-1}$ is the rate constant for ion-ion recombination; $q_{e}=1.62 \times 10^{-19} \mathrm{C}$ the electron charge; $\vec{U}$ is the velocity of air drifted by the motion of charge and $\epsilon_{0}=$ $8.854 \times 10^{-12} \mathrm{C} \cdot \mathrm{V}^{-1} \cdot \mathrm{m}^{-1}$ is the permittivity of the air. The electric field $\vec{E}$ is a function of the electric potential $\phi$ by the Gauss law $\vec{E}=\nabla \emptyset ; p$ the pressure, $\quad v=15.7 \times$ $10^{-3} \mathrm{~m}^{2} \cdot \mathrm{s}^{-1}$ the kinematic viscosity, and $\rho=$ $1.2041 \mathrm{~kg} \cdot \mathrm{m}^{-3}$ the air density; $i_{\exp }$ is determined by the experimental I-V characteristic; the charge density is applied to the area $A$ of the electrodes at which the electric field magnitude is greater than $E_{\text {on }}$ [49].

The solutions of Equations (2)-(6) with the boundary conditions were obtained using OpenFOAM based finite volume method [50]. The convection term $\vec{U} \rho_{ \pm}$in Equation (2) was neglected and an additional solver was developed to solve Equations (2) and (3) separately from Equations (4) and (5). The solution by Equations (2) and (3) was used as the initial electric field condition for solving the coupled Equations(1)-(4) in the main solver [24]. The simulation setup with the meshing and boundary conditions of the ion wind motion by the configuration SR400/SR15 is described in Figure 6.

Figure 7 presents the electric field distribution together with the ion winds generated by the configuration using electrodes of different tips. As expected, the ion wind is stronger with the electrode of smaller tip (SR15) and hence, the merging ion wind is deflected towards the electrode of round tip (SR400). The same observation can be found with different voltages as shown in Figure 8. However, the merging ion wind is less deflected towards the electrode of SR400 with higher discharge voltage. This observation is in good agreement with the experimental ion wind speed measured at different pin tips and with inter-electrode distances. The existence of optimum electrode distances in Figure 5, was not observed in the simulation range and will be investigated in our future work.

The asymmetry of ion wind generated by an asymmetric configuration is also demonstrated by directing the ion wind towards a mixture of di-propylene glycol monomethyl ether (DPM) and violet dye. Since the violet dye is partly dissolved in the DPM, the mixture can be clearly observed once the violet die is redistributed under the effect of ion wind.

Experiment shows that when the ion wind is placed close to the mixture, the movement of violet dye is observed (Figure 9a). After a few seconds, the violet dye is redistributed and two blank regions are formed (Figure 9bd). Both of them are just underneath the electrodes, while the fluid rotates in the clockwise direction. Figure $9 \mathrm{e}$ and $\mathrm{f}$ clearly depicts that the two blank regions are difference in size caused by the dual vortices, one is stronger than another as observed in Figure $9 \mathrm{f}-\mathrm{h}$. The work also demonstrates the potential of bipolar discharge ion wind generator using 
asymmetric configurations in mixing applications.

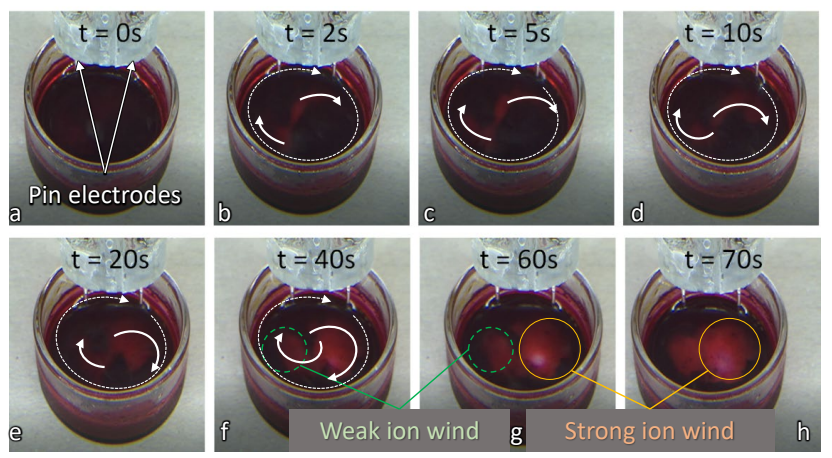

Figure 9. Illustration of a mixture using the proposed device.

\section{CONCLUSION}

In this work, we have further studied bipolar ion wind generator using asymmetric configurations of parallel electrodes where both of them generate corona discharge. A combination of electrodes of different pin tips has been installed to create asymmetrical ion wind. The system was investigated and evaluated by both experimental and simulation works. The research allows to predict different operation regimes of generator using asymmetric electrodes to generate ion wind based on the I-V characteristics and ion wind speed measured by experiments. An optimization of the configuration can generate ion wind flowing up to $2.24 \mathrm{~m} / \mathrm{s}$ at the atmospheric pressure. Furthermore, the asymmetric configuration with different tip shapes can be used in many mixing applications such as the mixing solvent and die which was demonstrated in this work.

\section{ACKNOWLEDGEMENT}

Tung Thanh Bui would like to thank to Vietnam National Foundation for Science and Technology Development (NAFOSTED) for its financial support under grant 107.992016.36

\section{REFERENCES}

[1] A. P. Chattock, "On the velocity and mass of the ions in the electric wind in air," Philos. Mag. Ser. 5, vol. 48, no. 294, pp. 401-420, Nov. 1899.

[2] A. A. Martins, "Modelling of an improved positive corona thruster and actuator," J. Electrostat., vol. 71, no. 1, pp. 61-67, 2013.

[3] L. Zhao and K. Adamiak, "Numerical analysis of forces in an electrostatic levitation unit," J. Electrostat., vol. 63, no. 6-10, pp. 729-734, 2005.

[4] A. A. Martins and M. J. Pinheiro, "On the influence that the ground electrode diameter has in the propulsion efficiency of an asymmetric capacitor in nitrogen gas," Phys. Plasmas, vol. 18, no. 3, p. 33512, Mar. 2011.

[5] E. Moreau, "Airflow control by non-thermal plasma actuators," J. Phys. D. Appl. Phys., vol. 40, no. 3, pp. 605-636, Feb. 2007.

[6] Jae-Duk Moon, Deok-hyun Hwang, and Sang-Taek Geum, “An EHD gas pump utilizing a ring/needle electrode," IEEE Trans. Dielectr. Electr. Insul., vol. 16, no. 2, pp. 352-358, Apr. 2009.

[7] O. M. Stuetzer, "Ion drag pumps," J. Appl. Phys., vol. 31, no. 1, pp. 136-146, 1960.
[8] M. Rickard, D. Dunn-Rankin, F. Weinberg, and F. Carleton, "Characterization of ionic wind velocity," J. Electrostat., vol. 63, no. 6-10, pp. 711-716, 2005.

[9] W. Wang, L. Yang, P. Huo, and K. Wu, "Thrust characteristic of a multipoint-grid type EHD gas pump under negative corona operation in air," IEEE Trans. Dielectr. Electr. Insul., vol. 22, no. 4, pp. 21362141, 2015.

[10] V. A. Chirkov, Y. K. Stishkov, and S. A. Vasilkov, "Characteristics of electrohydrodynamic pump of the dissociation type: Low and high voltage ranges," IEEE Trans. Dielectr. Electr. Insul., vol. 22, no. 5, pp. 2709-2717, 2015.

[11] D. B. Go, T. S. Fisher, and S. V Garimella, "Direct simulation of ionization and ion transport for planar microscale ion generation devices," J. Phys. D. Appl. Phys., vol. 42, no. 5, p. 55203, 2009.

[12] K. G. Kibler and H. G. Carter, "Electrocooling in gases," J. Appl. Phys., vol. 45, no. 10, pp. 4436-4440, 1974.

[13] A. Rashkovan, E. Sher, and H. Kalman, "Experimental optimization of an electric blower by corona wind," Appl. Therm. Eng., vol. 22, no. 14 , pp. 1587-1599, 2002

[14] D. F. Colas, A. Ferret, D. Z. Pai, D. a. Lacoste, and C. O. Laux, "Ionic wind generation by a wire-cylinder-plate corona discharge in air at atmospheric pressure," J. Appl. Phys., vol. 108, no. 10, pp. 0-6, 2010.

[15] D. I. Carroll, I. Dzidic, R. N. Stillwell, K. D. Haegele, and E. C. Horning, "Atmospheric pressure ionization mass spectrometry. Corona discharge ion source for use in a liquid chromatograph-mass spectrometer-computer analytical system," Anal. Chem., vol. 47, no. 14, pp. 2369-2373, 1975.

[16] G. F. L. Ferreira, O. N. Oliveira, and J. a. Giacometti, "Point-to-plane corona: Current-voltage characteristics for positive and negative polarity with evidence of an electronic component," J. Appl. Phys., vol. 59, no. 9, pp. 3045-3049, 1986.

[17] E. Moreau and G. Touchard, "Enhancing the mechanical efficiency of electric wind in corona discharges," J. Electrostat., vol. 66, no. 12, pp. 39-44, 2008.

[18] H. Kawamoto and S. Umezu, "Electrostatic micro-ozone fan that utilizes ionic wind induced in pin-to-plate corona discharge system," J. Electrostat., vol. 66, no. 7-8, pp. 445-454, 2008.

[19] D. B. Go, S. V. Garimella, T. S. Fisher, and R. K. Mongia, "Ionic winds for locally enhanced cooling," J. Appl. Phys., vol. 102, no. 5, 2007

[20] C. Kim, K. C. Noh, J. Hyun, S. G. Lee, J. Hwang, and H. Hong, "Microscopic energy conversion process in the ion drift region of electrohydrodynamic flow," Appl. Phys. Lett., vol. 100, no. 24, 2012.

[21] M. Ghazanchaei, K. Adamiak, and G. S. S. P. Castle, "Predicted flow characteristics of a wire-nonparallel plate type electrohydrodynamic gas pump using the Finite Element Method," J. Electrostat., vol. 73, pp. 103-111, 2015.

[22] V. T. Dau, T. X. Dinh, T. T. Bui, and T. Terebessy, "Bipolar corona assisted jet flow for fluidic application," Flow Meas. Instrum., vol. 50, pp. 252-260, 2016.

[23] V. T. Dau, T. X. Dinh, T. T. Bui, C. D. Tran, H. T. Phan, and T. Terebessy, "Corona based air-flow using parallel discharge electrodes,” Exp. Therm. Fluid Sci., vol. 79, pp. 52-56, 2016.

[24] V. T. Dau, T. X. Dinh, T. Terebessy, and T. T. Bui, "Ion Wind Generator Utilizing Bipolar Discharge in Parallel Pin Geometry," IEEE Trans. Plasma Sci., vol. 44, no. 12, pp. 2979-2987, Dec. 2016.

[25] V. T. Dau, T. X. Dinh, T. Terebessy, and T. T. Bui, "Bipolar corona discharge based air flow generation with low net charge," Sensors Actuators A Phys., vol. 244, pp. 146-155, 2016.

[26] T. X. Dinh, D. B. Lam, C.-D. Tran, T. T. Bui, P. H. Pham, and V. T. Dau, "Jet flow in a circulatory miniaturized system using ion wind," Mechatronics, vol. 47, no. September, pp. 126-133, Nov. 2017.

[27] V. T. Dau, T. X. Dinh, T. T. Bui, and T. Terebessy, "Corona anemometry using dual pin probe," Sensors Actuators A Phys., vol. 257, pp. 185-193, 2017.

[28] V. T. Dau, T. T. Bui, T. X. Dinh, and T. Terebessy, "Pressure sensor based on bipolar discharge corona configuration," Sensors Actuators A Phys., vol. 237, pp. 81-90, Jan. 2016.

[29] P. K. Wong, T.-H. Wang, J. H. Deval, and C.-M. Ho, "Electrokinetics in Micro Devices for Biotechnology Applications," IEEE/ASME Trans. Mechatronics, vol. 9, no. 2, pp. 366-376, Jun. 2004.

[30] S. J. Liu, H. H. Wei, S. H. Hwang, and H. C. Chang, "Dynamic particle trapping, release, and sorting by microvortices on a 
substrate," Phys. Rev. E - Stat. Nonlinear, Soft Matter Phys., vol. 82, no. 2,2010 .

[31] F. Weinberg, F. Carleton, D. Kara, A. Xavier, D. Dunn-Rankin, and M. Rickard, "Inducing gas flow and swirl in tubes using ionic wind from corona discharges," Exp. Fluids, vol. 40, no. 2, pp. 231-237, 2006.

[32] L. Y. Yeo and J. R. Friend, "Electrohydrodynamic Flow for Microfluidic Mixing and Microparticle Manipulation," in International Symposium on Electrohydrodynamics (ISEHD), Buenos Aires Argentina, 2014, no. December, pp. 4-7.

[33] L. Y. L. Yeo, D. Hou, S. Maheshswari, and H.-C. C. Chang, "Electrohydrodynamic surface microvortices for mixing and particle trapping,” Appl. Phys. Lett., vol. 88, no. 23, pp. 2006-2008, 2006.

[34] D. Hou, S. Maheshwari, and H. C. Chang, "Rapid bioparticle concentration and detection by combining a discharge driven vortex with surface enhanced Raman scattering," Biomicrofluidics, vol. 1, no. 1, 2007.

[35] P. Zhao, S. Portugal, and S. Roy, "Efficient needle plasma actuators for flow control and surface cooling," Appl. Phys. Lett., vol. 107, no. 3, p. 33501, 2015.

[36] V. Scholtz, J. Pazlarova, H. Souskova, J. Khun, and J. Julak, "Nonthermal plasma - A tool for decontamination and disinfection," Biotechnol. Adv., vol. 33, no. 6, pp. 1108-1119, 2015.

[37] F. Shen, W. Kai, and M. Yao, "Negatively and positively charged bacterial aerosol concentration and diversity in natural environments," Chinese Sci. Bull., vol. 58, no. 26, pp. 3169-3176, Jun. 2013.

[38] K. Wei, Z. Zou, and M. Yao, "Charge levels and Gram (+/-) fractions of environmental bacterial aerosols," J. Aerosol Sci., vol. 74, pp. 5262, 2014.

[39] W. L. Lama and C. F. Gallo, "Systematic study of the electrical characteristics of the 'trichel' current pulses from negative needle-toplane coronas," J. Appl. Phys., vol. 45, no. 1, pp. 103-113, 1974.

[40] X. Meng, H. Zhang, and J. (Jesse) Zhu, "A general empirical formula of current-voltage characteristics for point-to-plane geometry corona discharges," J. Phys. D. Appl. Phys., vol. 41, no. 6, p. 65209, 2008.

[41] B. Kim, S. Lee, Y. S. Lee, and K. H. Kang, "Ion wind generation and the application to cooling," J. Electrostat., vol. 70, no. 5, pp. 438444, 2012.

[42] B. L. Henson, "A space-charge region model for microscopic steady coronas from points," J. Appl. Phys., vol. 52, no. 2, pp. 709-715, 1981.

[43] N. E. Jewell-Larsen, C. P. Hsu, I. a. Krichtafovitch, S. W. Montgomery, J. T. Dibene, and A. V. Mamishev, "CFD analysis of electrostatic fluid accelerators for forced convection cooling," IEEE Trans. Dielectr. Electr. Insul., vol. 15, no. 6, pp. 1745-1753, 2008.

[44] V. A. Chirkov, Y. K. Stishkov, and A. A. Sitnikov, "Simulation of the integral electric current characteristics of unsteady-state current passage through liquid dielectrics," IEEE Trans. Dielectr. Electr. Insul., vol. 22, no. 5, pp. 2763-2769, 2015.

[45] J. Shrimpton and A. Kourmatzis, "Direct numerical simulation of forced flow dielectric EHD within charge injection atomizers," IEEE Trans. Dielectr. Electr. Insul., vol. 17, no. 6, pp. 1838-1845, 2010.

[46] Z. M. Al-hamouz, "Adaptive finite-element ballooning analysis of bipolar ionized fields," IEEE Trans. Ind. Appl., vol. 32, no. 6, pp. 1266-1277, 1996.

[47] D. Cagnoni, F. Agostini, T. Christen, N. Parolini, I. Stevanović, and C. De Falco, "Multiphysics simulation of corona discharge induced ionic wind," J. Appl. Phys., vol. 114, no. 233301, 2013.

[48] K. Adamiak, "Numerical models in simulating wire-plate electrostatic precipitators: A review," J. Electrostat., vol. 71, no. 4, pp. 673-680, 2013.

[49] J. S. Chang, P. a. Lawless, and T. Yamamoto, "Corona discharge processes,” IEEE Trans. Plasma Sci., vol. 19, no. 6, pp. 1152-1166, 1991.

[50] OpenFOAM $®$, "OpenFOAM ${ }^{\circledR} \mid$ The OpenFOAM Foundation," 2016. [Online]. Available: http://openfoam.org/.

Van Thanh Dau received the B.S. degree in aerospace engineering from Hochiminh City University of Technology, Vietnam, in 2002, and the M.Sc. and $\mathrm{Ph} . \mathrm{D}$. degrees in micro-mechatronics from Ritsumeikan University, Japan, in 2004 and 2007, respectively. He has been with the Research Group, Sumitomo Chemical, Japan since 2010, exploring the integrated micro fluidic atomization methods. He has authored over 100 papers in scientific journals and conference proceeding. He has filed over 30 intellectual properties, been granted seven US and many international patents. His research interests include microfluidics, sensors and actuators, nanoparticle generation and electro-fluido-dynamic.

Dr. Dau was a recipient of the Japan Society for the Promotion of Science Post-Doctoral Fellowship from 2007 to 2009. He was a visiting scholar at the National Institute of Advanced Industrial Science and Technology, Japan in 2009, and a visiting researcher at Atrium Innovation, Ltd. Oxfordshire, United Kingdom in 2011.

Canh-Dung Tran (CD Tran) is currently working as a Senior Lecturer at School of Mechanical and Electrical Engineering, Faculty of Health, Engineering and Sciences, Senior Research Fellow of the Computational Engineering Scientific Research Centre, The University of Southern Queensland (USQ), Australia; and Research Fellow at the Department of Mechanical Engineering, National university of Singapore. From 2005 to 2010, he was a Research Scientist and Project Leader at the CSIRO and participated in various projects relating to modelling and analyzing the mechanical behaviour of materials. He has gained wide experience in developing mechanical, material engineering and Carbon Nano-tube research proposals, which were funded internally and externally, in conjunction with the CSIRO, University of Texas at Dallas (USA), University of Sydney (Australia) and the University College of London (UK). Supported by a USQ scholarship, he earned his $\mathrm{PhD}$ degree in Mechanical Engineering in 2004. In 1994, he received a national scholarship of the French Government to complete his research DEA program on Mechanics of Advanced Fibrous Materials at the University of Haut Alsace, France. He has produced about 100 research publications as author and coauthors in the areas of numerical simulation including stochastic macromicro methods, advanced Fibrous Materials, nanomaterials and CNTs.

Thien Xuan Dinh received the B.S. degree in aerospace engineering from Hochiminh City University of Technology in 2002, Vietnam and the M.Sc. and Ph.D. degrees in mechanical engineering from Ritsumeikan University in 2004 and 2007, respectively. He was recipient of Japan Government Scholarship (MEXT) for Outstanding Student to pursuits his M. Sc. and Ph. D. courses and Japan Society for the Promotion of Science postdoctoral fellowship from 2011 to 2013.

His general research interest is computation of fluid flow. The large parts of his research are turbulence modeling using Large Eddy Simulation, multiphase modeling using Volume of Fluid technique, and simulation of turbulence and dispersion. Recently, he has focused on computation of fluid flow for developing microfluidic devices as electro-hydro-dynamics, microsensors, micro-pump, and micro-mixer for biochemical engineering.

Dang Bao Lam received the Eng. degree from Slovak University of Technology (1998), the M.E. degree in Mechanics of Machines (2004), and the D.Eng. degree in MEMS engineering from Hanoi University of Science and Technology (2015). He has been a lecturer in a School of Mechanical Engineering, HUST since 2001. His current interests include MEMS based sensors, actuators and applications.

Tibor Terebessy received his M.S. degree with honour in plasma physics from Comenius University, Slovakia, in 1998 and his Ph.D. degree in electronics engineering from Shizuoka University, Japan, in 2002. He was then awarded a Postdoctoral Fellowship by the Japan Society for the Promotion of Science (JSPS), continuing his research in large area microwave discharges and their industrial applications at Graduate School of Electronic Science and Technology, Shizuoka University, Japan.

His main areas of research interests include atmospheric pressure discharges, microwave plasmas, nanoparticle generation and electrohydrodynamics. He is the author and co-author of more than 20 scientific articles and 17 inventions.

Bui Thanh Tung received the B.S. degree in electrical engineering from Vietnam National University, Hanoi (VNUH) in 2004, and the M.E. and D.Eng. degrees in Science and Engineering from Ritsumeikan University, Shiga, Japan, in 2008 and 2011, respectively. From 2011 to 2015 he was a post-doctoral researcher with the 3D Integration System Group, Nanoelectronics Research Institute (NeRI), National Institute of Advanced Industrial Science and Technology (AIST), Tsukuba, Japan. Currently, he is an assistant professor at the Faculty of Electronics and Telecommunication (FET), University of Engineering and Technology (UET), Vietnam National 
University, Hanoi (VNUH). His current interests include MEMS based sensors, actuators and applications. 